\title{
Effect of temperature dependent viscosity on ferrothermohaline convection saturating an anisotropic porous medium with Soret effect using the Galerkin technique
}

\author{
K. Raju \\ Department of Mathematics, Achariya Arts and Science College, Villianur, Puducherry 605 110, India
}

Corresponding Author Email: rajumaths1987@gmail.com

https://doi.org/10.18280/ijht.360208

Received: 12 July 2017

Accepted: 28 April 2018

\section{Keywords:}

thermohaline convection, ferrofluid, anisotropy porous medium, Soret effect, brinkman model, temperature dependent viscosity, Galerkin technique

\begin{abstract}
In the present paper, the effect of temperature dependent viscosity on a Soret driven ferrothermohaline convection heated from below and salted from above subjected to a transverse uniform magnetic field in the presence of an anisotropic porous medium using Brinkman model is studied. For the case of two free boundaries, an exact solution is obtained using a linear stability analysis and normal mode technique is applied. The effect of salinity has been included in magnetization and density of the fluid. The critical thermal magnetic Rayleigh number $N_{\text {sc }}$ for the onset of instability is calculated numerically for sufficiently large values of the buoyancy magnetization parameter $M_{1}$ using the method of computational Galerkin technique. It is found that non-buoyancy magnetization parameter, permeability of the porous medium, anisotropy effect and temperature dependent viscosity stabilizes the system.
\end{abstract}

\section{INTRODUCTION}

For centuries, many fascinating materials have been attracting the scientists and researchers due to their extraordinary physical properties and technological usage. Magnetic fluids, also called 'ferrofluids', are electrically nonconducting colloidal suspensions of tiny particles of solid ferromagnetic material in a non-electrically conducting carrier fluid like water, hydrocarbon fuels, etc. These fluids behave as a homogeneous continuum and exhibit a variety of interesting phenomena. Ferromagnetic fluids are not found in nature but are artificially synthesized. The viscosity of a ferrofluid as a function of the applied magnetic field, direction of magnetic field with respect to the flow direction and temperature.

Ferrofluids are widely used in magnetic inkjet printers, heat transfer, nanomotors, nanogeneratores, inertial dampers, switches, sensors, transformer cooling, loudspeakers, microand nanofluidic devices, magnetic targeted drug delivery, etc. In the biomedical field, they have been found very useful. These can be used to deliver drugs to a certain area of human body and also used for cancer treatment by heating the tumor soaked in ferrofluids by means of an alternating magnetic field [1-3]. An authoritative introduction to the research on magnetic fluids has been given in the monograph by Rosensweig [4], which reviews several applications of heat transfer through ferrofluids, such as enhanced convective cooling having a temperature dependent magnetic moment due to magnetization of the fluid. This heat transfer through ferrofluids is called ferroconvection, which is similar to Bénard convection (Chandrasekhar [5]). Convective instability of ferromagnetic fluids has been predicted by Finlayson [6]. Schwab et al. [7] experimentally investigated Finlayson's problem under a strong magnetic field and detected the onset of convection by plotting the Nusselt number versus the magnetic Rayleigh number. Later, Stiles and Kagan [8] examined the experimental problem reported by Schwab et al. [7] and generalized Finlayson's model assuming that under a strong magnetic field, the rotational viscosity augments the shear viscosity.

In many investigations, porous medium is taken to be isotropic for geological and pedological process rarely it forms isotropic media, as is usually assumed in transport studies. Processes such as frost action, sedimentation, compaction and reorientation of solid matrix are responsible for the creation of anisotropic natural porous media. Ursino et al. [9] studied upscaling of anisotropy in unsaturated Miller-similar porous media. In this, analytical expressions for the anisotropic conductivity tensor are derived based on the dynamic law that governs the flow problem at the pore scale and the effects of anisotropy on transport parameters are estimated by numerical modeling. In chemical engineering processes, anisotropy can be characteristic of artificial porous like fiber materials. Epherre [10] was the first attempt to study the onset of convection in a horizontal porous layer with anisotropic thermal conductivity.

Vaidyanathan et al. [11] studied convective instability of ferromagnetic fluid in a porous medium of large permeability using Brinkman model. This investigation has been analyzed for the effect of temperature dependent viscosity by Ramanathan and Muchikel [12] using Galerkin technique. In this, temperature dependent viscosity is studied for stabilizing effect for different behaviors, which is not much pronounced. Govindan et al. [13] studied numerical analysis of ferroconvection with temperature dependent viscosity and an anisotropic porous medium. Nanjundappa et al. [14] introduced magnetic field dependent viscosity on MarangoniBénard ferroconvection without a porous medium under microgravity conditions in a horizontal ferrofluid layer in the presence of a uniform vertical magnetic field. This work has been analyzed to the effect temperature dependent viscosity in the absence of magnetic field dependent viscosity by Nanjundappa et al. [15]. They used the Rayleigh Ritz method 
with Chebyshev polynomials of second kind as trial function. Siddheshwar [16] studied the thermorheological effect of magnetoconvection in fluids with weak electrical conductivity.

The study of convection in two component ferrofluids will throw light on convective instability. This is referred to as a type of convection known as ferrothermohaline convection studied by Baines and Gill [17]. Vaidyanathan et al. [18-19] investigated the presence and absence of a porous medium on ferrothermohaline. Furthermore, Vaidyanathan et al. [20] attempted to study the Soret effect due to ferrothermohaline convection of a sparse distribution and the condition of a porous medium of ferroconvective instability of multicomponent fluid heated from below and salted from above was analyzed by Sekar et al. [21-22] for isotropic and anisotropic models. The effect of rotation on thermohaline convection in a ferromagnetic fluid saturating an anisotropic porous medium with Soret effect was obtained by Sekar et al. [23] and further investigation was carried out for magnetic field dependent viscosity by Sekar and Raju [24].

In the present work, it is attempted to analyze the effect of an anisotropic porous medium and temperature dependent viscosity on Soret driven ferrothermohaline convection, subjected to a vertical magnetic field using the Brinkman model and the free boundaries are considered. The resulting eigen value problem is solved numerically using the Galerkin method. Besides, an analytical formula is obtained for the critical magnetic Rayleigh number by a regular perturbation method.

\section{MATHEMATICAL FORMULATION}

An infinite spread horizontal layer of an OberbeckBoussinesq ferromagnetic fluid of thickness " $d$ " saturating a sparsely distributed anisotropic porous medium heated from below and salted from above is considered. The temperature and salinity at the bottom and top surfaces are $z= \pm d / 2$ $T_{0} \pm \Delta T / 2$ and $S_{0} \pm \Delta S / 2$, respectively. Both the boundaries are assumed to be free and perfect conductors of heat and salt. The system is assumed to be anisotropy along the vertical direction and isotropy along the horizontal direction and the fluid viscosity is assumed to be temperaturedependent in the following form $[16,12]$

$$
\mu(T)=\mu_{1}\left[1-\delta\left(T-T_{a}\right)^{2}\right]
$$

Considering the Soret effect on the temperature gradient the mathematical equations governing the above investigation are as follows with the porous medium $\mathrm{k}=\left(k_{1}, k_{1}, k_{2}\right)$.

The continuity equation for an incompressible fluid is

$$
\nabla \cdot \mathbf{q}=0
$$

The corresponding momentum equation is

$$
\rho_{0} \frac{D \mathbf{q}}{D t}=-\nabla p+\rho \mathbf{g}+\nabla \cdot(\mathbf{H B})+\nabla\left[\mu(T)\left(\nabla \mathbf{q}+\nabla \mathbf{q}^{T r}\right)\right]-\frac{\mu(\mathrm{T})}{k} \mathbf{q}
$$

The temperature equation for an incompressible $\left[\rho_{0} C_{v, H}-\mu_{o} \mathbf{H}(\partial \mathbf{M} / \partial T)_{v, H}\right](d T / d t)+\mu_{0} T(\partial \mathbf{M} / \partial T)_{v, H} \cdot(d \mathbf{H} / d t)=K_{1} \nabla^{2} T+\phi$

The conservation of mass flux equation is given by

$\rho_{0}\left(\partial / \partial t+\mathbf{q} ? S=K_{S} \nabla^{2} S+S_{T} \nabla^{2} T\right.$

The density equation of state for a Boussinesq twocomponent fluid is

$\rho=\rho_{0}\left[1-\alpha_{t}\left(T-T_{0}\right)+\alpha_{S}\left(S-S_{0}\right)\right]$

Maxwell's equations, simplified for a non-conducting fluid with no displacement currents, become

$\nabla \cdot \mathbf{B}=0, \quad \nabla \times \mathbf{H}=0$

where the magnetic induction is given by

$\mathbf{B}=\mu_{0}(\mathbf{M}+\mathbf{H})$

In general, the pressure of ferromagnetic fluid can distort an external magnetic field if magnetic interaction (dipole-dipole) takes place, but this is negligible for small particle concentration, as is assumed here. We assume that the magnetization is aligned with the magnetic field, but allow a dependence on the magnitude of the magnetic field, temperature and salinity, so that

$\mathbf{M}=\frac{\mathbf{H}}{H} M(H, T, S)$

The linearized magnetic equation is

$$
M=M_{0}+\chi\left(\mathrm{H}-H_{0}\right)-K\left(T-T_{0}\right)+K_{2}\left(S-S_{0}\right),
$$
by

The basic state is assumed to be quiescent state and is given

$$
\begin{aligned}
& \mathbf{q}=\mathbf{q}_{b}=(0,0,0), \quad T=T_{b}=T_{0}-\beta_{t} z, S=S_{b}=S_{0}-\beta_{S} z, \rho(z)=\rho_{0}\left[1+\alpha_{t} \beta_{t} z-\alpha_{S} \beta_{S} z\right] \\
& p=p_{b}(z), H_{b}(z)=\left[H_{0}-\frac{K \beta_{t} z}{1+\chi}+\frac{K_{2} \beta_{S} z}{1+\chi}\right] \mathbf{k}, \quad M_{b}(z)=\left[M_{0}+\frac{K \beta_{t} z}{1+\chi}-\frac{K_{2} \beta_{S} z}{1+\chi}\right] \mathbf{k} .
\end{aligned}
$$

Let the component of the perturbed magnetization and the magnetic field be $\left(M_{1}^{\prime}, M_{2}^{\prime}, M_{b}(z)+M_{3}^{\prime}\right)$ and $\left(H_{1}^{\prime}, H_{2}^{\prime}, H_{b}(z)+H_{3}^{\prime}\right)$, respectively. The perturbed viscosity and temperature are taken as $\mu_{b}(z)+\mu^{\prime}$ and $T_{b}(z)+T^{\prime}$, respectively. Moreover, the basic state is disturbed by an infinitesimal thermal perturbation and the basic state quantities are obtained by substituting the velocity of quiescent state in the governing Eqs. (1) - (4). The techniques of linearization and normal mode. [22,23] are used to finding the solutions of Eqs. (1) - (7). This can be written as

ferromagnetic fluid is 
$(w, T, \phi, S)=\left[w(z, t), T(z, t), \phi^{\prime}(z, t), S^{\prime}(z, t)\right] \exp \left(i\left(k_{x} x+k_{y} y\right)\right)$

The vertical component of momentum equation can be written as

$\rho_{0} \frac{\partial}{\partial t}\left(\frac{\partial^{2}}{\partial z^{2}}-k_{0}^{2}\right) w$

$=\rho_{0} g \alpha_{S} k_{0}^{2} S^{\prime}-\rho_{0} g \alpha_{t} k_{0}^{2} T^{\prime}+K \beta_{t} k_{0}^{2} \frac{\partial \phi^{\prime}}{\partial z}-\frac{\mu_{0} K^{2} k_{0}^{2} \beta_{t}\left(1-S_{T}\right)}{1+\chi} T^{\prime}+\frac{\mu_{0} K K_{2} \beta_{S} k_{0}^{2}\left(1-S_{T}\right)}{1+\chi} T^{\prime}$

$+\frac{\mu_{0} K K_{2} \beta_{t} k_{0}^{2}}{1+\chi} S^{\prime}-\frac{\mu_{0} K^{2} k_{0}^{2} \beta_{S}}{1+\chi} S^{\prime}+\mu_{0}\left(\frac{\partial^{2}}{\partial z^{2}}-k_{0}^{2}\right)^{2} w+\mu_{0} K_{2} \beta_{S} k_{0}^{2} \frac{\partial \phi^{\prime}}{\partial z}+\frac{\mu_{b}}{k_{1}} k_{0}^{2} w$

$+\frac{\partial^{2} \mu_{b}}{\partial z^{2}}\left(\frac{\partial^{2}}{\partial z^{2}}-k_{0}^{2}\right) w+2 \frac{\partial \mu_{b}}{\partial z} \frac{\partial}{\partial z}\left(\left(\frac{\partial^{2}}{\partial z^{2}}-k_{0}^{2}\right) w\right)-\frac{1}{k_{2}} \frac{\partial \mu_{b}}{\partial z}\left(\frac{\partial w}{\partial z}\right)$

The modified Fourier heat conduction equation is

$\rho_{0} C_{v, H} \frac{\partial \theta}{\partial t}-\mu_{0} K T_{0} \frac{\partial}{\partial t}\left(\frac{\partial \phi}{\partial z}\right)=K_{1}\left(\frac{\partial^{2}}{\partial z^{2}}-k_{0}^{2}\right) \theta+\left[\rho_{0} c \beta_{t}-\left(\frac{\mu_{0} K^{2} T_{0}^{2} \beta_{t}}{1+\chi}\right)+\left(\frac{\mu_{0} K K_{2} T_{0} \beta_{S}}{1+\chi}\right)\right] w$,

where $\rho_{0} C=\rho_{0} C_{v, H}+\rho_{0} K H_{0}$.

The salinity equation is

$\frac{\partial S}{\partial t}+\beta_{S} w=K_{S}\left(\frac{\partial^{2}}{\partial z^{2}}-k_{0}^{2}\right) S+S_{T}\left(\frac{\partial^{2}}{\partial z^{2}}-k_{0}^{2}\right) \theta$

Using the analysis similar to Sekar et al. [23] one gets

$(1+\chi) \frac{\partial^{2} \phi}{\partial z^{2}}+\left(1+\frac{M_{0}}{H_{0}}\right) k_{0}^{2} \phi-K \frac{\partial \theta}{\partial z}+K_{2} \frac{\partial S}{\partial z}+S_{T} K \frac{\partial \theta}{\partial z}=0$.

where $\nabla_{1}^{2}=\left(\partial^{2} / \partial x^{2}\right)+\left(\partial^{2} / \partial y^{2}\right)$ and $\nabla^{2}=\nabla_{1}^{2}+\left(\partial^{2} / \partial z^{2}\right)$.

The non-dimensional numbers can be written using

$w^{*}=\frac{w d}{v}, t^{*}=\frac{v t}{d^{2}}, T^{*}=\left(\frac{K_{1} a R^{1 / 2}}{\rho_{0} C_{v, H} \beta_{t} v d}\right) \theta, \quad \phi^{*}=\left(\frac{(1+\chi) K_{1} a R^{1 / 2}}{\rho_{0} C_{v, H} K \beta_{t} v d^{2}}\right) \phi, \quad z^{*}=\frac{z}{d}, \quad a=k_{0} d$,

$D=\frac{\partial}{\partial z^{*}}, S^{*}=\left(\frac{K_{S} a R_{S}^{1 / 2}}{\rho_{0} C_{v, H} \beta_{S} v d}\right) S, \quad v=\frac{\mu}{\rho_{0}}, \quad k^{*}=\frac{k}{d^{2}}, M_{1}=\frac{\mu_{0} K^{2} \beta_{t}}{(1+\chi) \rho_{0} g \alpha_{t}}, \quad M_{2}=\frac{\mu_{0} K^{2} T}{(1+\chi) \rho_{0} C_{v, H}}$,

$M_{3}=\frac{1+\left(M_{0} / H_{0}\right)}{(1+\chi)}, M_{4}=\frac{\mu_{0} K^{2} \beta_{S}}{(1+\chi) \rho_{0} g \alpha_{S}}, M_{5}=\frac{K_{2} \beta_{S}}{K \beta_{t}}, M_{6}=\frac{K_{S}}{K_{1}} P_{r}=\frac{\mu C_{v, H}}{K_{1}}$,

$R=\frac{\rho_{0} C_{v, H} \beta_{t} \alpha_{t} g d^{4}}{v K_{1}}, R_{S}=\frac{\rho_{0} C_{v, H} \beta_{S} \alpha_{S} g d^{4}}{v K_{S}}, \tau=\rho_{0} C_{v, H}\left(\frac{K_{S}}{K_{1}}\right)$

where $R$ is the thermal Rayleigh number, $R_{S}$ is the salinity Rayleigh number, $P_{r}$ is the Prandtl number.

Then the Eqs. (13) - (16) become

$$
\begin{aligned}
\frac{\partial}{\partial t^{*}}\left(D^{2}-a^{2}\right) w^{*}= & a R^{1 / 2}\left[M_{1} D \phi^{*}-\left(1+M_{1}\left(1-S_{T}\right) T^{*}\right)\right]+M_{1} M_{5} a R^{1 / 2} D \phi^{*}-M_{1} M_{5} a R^{1 / 2}\left(1-S_{T}\right) T^{*} \\
& +\left(D^{2}-a^{2}\right)^{2} w^{*}+a R_{S}^{1 / 2}\left[1+M_{4}+M_{4} M_{5}^{-1}\right] S^{*}+\left(1-V z^{* 2}\right)\left(D^{2}-a^{2}\right)^{2} w^{*} \\
& -\frac{\left(1-V z^{2}\right)}{k^{*}}\left(D^{2}-a^{2}\right) w^{*}-2 V\left(D^{2}-a^{2}\right) w^{*}-4 V z^{*}\left(D^{2}-a^{2}\right) D w^{*}+\frac{2 V z^{*}}{k^{*}} D w^{*}
\end{aligned}
$$

$P_{r}\left[\frac{\partial T^{*}}{\partial t^{*}}-M_{2} \frac{\partial}{\partial t^{*}}\left(D \phi^{*}\right)\right]=\left(D^{2}-a^{2}\right) T^{*}+a R^{1 / 2}\left(1-M_{2}-M_{2} M_{5}\right) w^{*}$,

$$
P_{r} \frac{\partial S^{*}}{\partial t^{*}}=\tau\left(D^{2}-a^{2}\right) S^{*}-a R_{S}^{1 / 2} M_{6} w^{*}+S_{T} M_{5} M_{6}^{-1}\left(R / R_{S}\right)^{1 / 2}\left(D^{2}-a^{2}\right) T^{*},
$$

$$
D^{2} \phi^{*}-M_{3} a^{2} \phi^{*}-\left(1-S_{T}\right) D T^{*}+M_{5} M_{6}^{-1}\left(R / R_{S}\right)^{1 / 2} D S^{*}=0,
$$

where the following non-dimensional parameters are introduced.

\section{EXACT SOLUTION FOR FREE BOUNDARIES USING GALERKIN TECHNIQUE}

The simplest boundary conditions chosen, namely free-free, isothermal with infinite magnetic susceptibility $\chi$ in the perturbed field keep the problem analytically tractable and serve the purpose of providing a qualitative insight into the problem. The case of two free boundaries is of little physical interest, but it is mathematically important because one can derive an exact solution, whose properties guide our analysis. Thus the exact solution of the system subjected to the boundary conditions

$w^{*}=D^{2} w^{*}=T^{*}=D \phi^{*}=S^{*}=0$ at $z^{*}=-1 / 2$ and

$z^{*}=+1 / 2$

is written in the form

$$
\begin{aligned}
& w^{*}=A w_{1}(z) e^{\sigma t^{*}} \cos \pi z^{*}, \quad T^{*}=B T_{1}(z) e^{\sigma t^{*}} \cos \pi z^{*}, \quad S^{*}=C S_{1}(z) e^{\sigma t^{*}} \cos \pi z^{*} \\
& D \phi^{*}=F \phi_{1}(z) e^{\sigma t^{*}} \cos \pi z^{*}, \quad \phi^{*}=\frac{F}{\pi} \phi_{1}(z) e^{\sigma t^{*}} \sin \pi z^{*}
\end{aligned}
$$

Substituting Eq. (23) in linearized perturbation dimensionless equations (Eqs 18-21), we get the following equations

$$
\begin{aligned}
& \left\{\begin{array}{l}
\sigma\left(D^{2}-a^{2}\right)^{2} w_{1}(z)-\left(1-V z^{2}\right)\left(D^{2}-a^{2}\right)^{2} w_{1}(z) \\
+2 V\left(D^{2}-a^{2}\right)\left[1+2 z D w_{1}(z)\right]+\left(\frac{\left(1-V z^{2}\right) \mathrm{a}^{2}}{k_{1}}\right) w_{1}(z)-\frac{1}{k_{2}} 2 v z D w_{1}(z)
\end{array}\right\} A \\
& +a R^{1 / 2}\left\{1+M_{1}\left(1+M_{5}\right)\left(1-S_{T}\right) T_{1}(z)\right\} B-a R_{S}^{1 / 2}\left(1+M_{4}+M_{4} M_{5}^{-1}\right) S_{1}(z) C \\
& +a R^{1 / 2} M_{1}\left(1+M_{5}\right) D \phi_{1}(z) F=0
\end{aligned}
$$

$a R^{1 / 2}\left(1-M_{2}-M_{2} M_{5}\right) w_{1}(z) A+\left(D^{2}-a^{2}-P_{r} \sigma\right) T_{1}(z) B+P_{r} \sigma M_{2} D \phi_{1}(z) F=0$,

$-a R_{S}^{1 / 2} M_{6} w_{1}(z) A+S_{T} M_{5} M_{6}^{-1}\left(\frac{R}{R_{S}}\right)^{1 / 2}\left(D^{2}-a^{2}\right) T_{1}(z) B+\left\{\tau\left(D^{2}-a^{2}\right)-\sigma P_{r}\right\} S_{1}(z) C=0$,

$-R_{S}^{1 / 2} \pi^{2}\left(1-S_{T}\right) D T_{1}(z) B+R^{1 / 2} M_{5} M_{6}^{-1} D S_{1}(z) C+R_{S}^{1 / 2}\left(D^{2}-a^{2} M_{3}\right) \phi_{1}(z) F=0$, 
For existence of non-trivial solutions, the determine of the coefficients of $A, B, C$ and $F$ must vanish. This determinant on simplification yields

$X \sigma^{3}-Y \sigma^{2}+Z \sigma+W=0$.

where,

$$
\begin{aligned}
& X=-c_{5} P_{r}\left\langle S_{1}\left\langle P_{r} T_{1} c_{1} w_{1}\right\rangle \phi_{1}\right\rangle \\
& Y=c_{1} c_{5}\left\langle T_{1}\left\langle P_{r} S_{1} c_{1} w_{1}\right\rangle \phi_{1}\right\rangle-c_{5} P_{r} \tau c_{1}\left\langle T_{1}\left\langle S_{1} w_{1}\right\rangle \phi_{1}\right\rangle \\
& Z=+\frac{a^{2}}{k_{1}} c_{5}\left\{\left\langle\phi_{1}\left\langle P_{r} T_{1} \tau c_{1} S_{1}\right\rangle\left(1-V z^{2}\right) w_{1}\right\rangle-\left\langle\phi_{1}\left\langle c_{1} T_{1} P_{r} S_{1}\right\rangle\left(1-V z^{2}\right) w_{1}\right\rangle\right\} \\
& +\frac{2}{k_{2}} c_{5}\left\{\left\langle V z D w_{1}\left\langle c_{1} T_{1} P_{r} S_{1}\right\rangle c_{5} \phi_{1}\right\rangle-\left\langle D w_{1}\left\langle P_{r} T_{1} \tau c_{1} S_{1}\right\rangle \phi_{1}\right\rangle\right\}-c_{1}^{2} c_{5}\left\langle\left(1-V z^{2}\right) w_{1}\left\langle P_{r} T_{1} \quad \tau b_{1} S_{1}\right\rangle \phi_{1}\right\rangle \\
& a^{2} R\left\langle c_{2} D \phi_{1}\left\langle\left(1-S_{T}\right) D T_{1} w_{1}\right\rangle P_{r} S_{1}\right\rangle-c_{1} c_{5}\left\langle T_{1}\left\langle c_{1} w_{1} \quad \tau c_{1} S_{1}\right\rangle \phi_{1}\right\rangle+a^{2} R c_{2} c_{4}\left\langle D \phi_{1}\left\langle P_{r} T_{1} M_{6} w_{1}\right\rangle D S_{1}\right\rangle \\
& +a^{2} R_{S} c_{3} c_{5}\left\langle S_{1}\left\langle P_{r} T_{1} M_{6} w_{1}\right\rangle \phi_{1}\right\rangle+\left\langle\left(1-V z^{2}\right) c_{1}^{2} w_{1}\left\langle c_{1} T_{1} P_{r} S_{1}\right\rangle c_{5} \phi_{1}\right\rangle \\
& -\left\langle 2 V c_{1}\left(1+2 z D w_{1}\right)\left\langle c_{1} T_{1} P_{r} S_{1}\right\rangle c_{5} \phi_{1}\right\rangle+a^{2} R\left\langle\left(1+c_{2}\left(1-S_{T}\right) T_{1}\right)\left\langle w_{1} P_{r} S_{1}\right\rangle c_{5} \phi_{1}\right\rangle \\
& +c_{1} c_{5}\left\langle\phi_{1}\left\langle P_{r} T_{1} \quad \tau c_{1} S_{1}\right\rangle 2 V\left(1+2 z D w_{1}\right)\right\rangle \\
& W=a^{2} R c_{4} c_{2}\left\langle D \phi_{1}\left\langle w_{1} S_{T} c_{1} c_{4} T_{1}\right\rangle D S_{1}\right\rangle+c_{1}^{2} c_{5}\left\langle\left(1-V z^{2}\right) w_{1}\left\langle c_{1} T_{1} \tau c_{1} S_{1}\right\rangle \phi_{1}\right\rangle \\
& -\left\langle 2 V c_{1}\left(1+2 z D w_{1}\right)\left\langle c_{1} T_{1} \tau c_{1} S_{1}\right\rangle c_{5} \phi_{1}\right\rangle \\
& +\frac{2}{k_{2}}\left\langle V z D w_{1}\left\langle c_{1} T \quad c_{5} \phi_{1}\right\rangle \tau c_{1} S_{1}\right\rangle-\frac{a^{2}}{k_{1}} c_{5}\left\langle\left(1-V z^{2}\right) w_{1}\left\langle c_{1} T_{1} \quad \tau c_{1} S_{1}\right\rangle \phi_{1}\right\rangle \\
& +a^{2} R c_{5}\left\langle\left(1+c_{2}\left(1-S_{T}\right) T_{1}\right)\left\langle w_{1} \quad \tau c_{1} S_{1}\right\rangle \phi_{1}\right\rangle+a^{2} R_{S}\left\langle c_{3} S_{1}\left\langle w_{1} S_{T} c_{1} c_{4} T_{1}\right\rangle c_{5} \phi_{1}\right\rangle \\
& +a^{2} R \tau c_{1} c_{2}\left\langle D \phi_{1}\left\langle w_{1}\left(1-S_{T}\right) D T_{1}\right\rangle S_{1}\right\rangle \\
& +a^{2} R c_{2} c_{4}\left\langle D \phi_{1}\left\langle c_{1} T_{1} M_{6} w_{1}\right\rangle D S_{1}\right\rangle+a^{2} R_{S} c_{3} c_{5}\left\langle S_{1}\left\langle P_{r} T_{1} M_{6} w_{1}\right\rangle \phi_{1}\right\rangle
\end{aligned}
$$

For obtaining stationary instability, the time-dependent term $T_{4}$ is equal to zero. From Eq. (28) it is easy to obtain the eigenvalue $R_{s c}$ and upon using $k_{2}=\varepsilon k_{1}$, where $\varepsilon$ is non dimensional parameter governing anisotropy.

$$
R_{s c}=\frac{x_{1}-a^{2} R_{S}\left(x_{2} S_{T}+x_{3}\right)}{\tau x_{4}+M_{1}\left(1+M_{5}\right)\left[\left(1-S_{T}\right) \tau x_{5}+x_{6} S_{T}+\tau\left(1-S_{T}\right) x_{7}+x_{8}\right]}
$$

where

$$
\begin{aligned}
& x_{1}=\left\langle\left(1-V z^{2}\right) c_{1}^{2} w_{1}\left\langle\tau c_{1} S_{1} \quad c_{1} T_{1}\right\rangle c_{5} \phi_{1}\right\rangle-\frac{a^{2}}{k_{1}}\left\langle\left(1-V z^{2}\right) w_{1}\left\langle c_{1} T_{1} \quad \tau c_{1} S_{1}\right\rangle c_{5} \phi_{1}\right\rangle \\
& +\frac{2}{\varepsilon k_{1}}\left\langle V z D w_{1}\left\langle c_{1} T \quad c_{5} \phi_{1}\right\rangle \tau c_{1} S_{1}\right\rangle-\left\langle c_{5} \phi_{1}\left\langle c_{1} T_{1} \quad \tau c_{1} S_{1}\right\rangle \quad 2 V c_{1}\left(1+2 z D w_{1}\right)\right\rangle \\
& x_{2}=c_{3} c_{5}\left\langle\phi _ { 1 } \left\langle\begin{array}{lll}
w_{1} & \left.\left.c_{1} c_{4} T_{1}\right\rangle S_{1}\right\rangle
\end{array}\right.\right. \\
& x_{3}=c_{3} c_{5}\left\langle S_{1}\left\langle P_{r} T_{1} M_{6} w_{1}\right\rangle \phi_{1}\right\rangle \\
& x_{4}=\left\langle 1\left\langle w_{1} \tau c_{1} S_{1}\right\rangle c_{5} \phi_{1}\right\rangle \\
& x_{5}=\tau\left\langle c_{1} S_{1}\left\langle c_{5} \phi_{1} T_{1}\right\rangle w_{1}\right\rangle \\
& x_{6}=c_{4}\left\langle D S_{1}\left\langle w_{1} c_{1} c_{4} T_{1}\right\rangle D \phi_{1}\right\rangle \\
& x_{7}=c_{1}\left\langle\begin{array}{lll}
D \phi_{1} & \left\langle w_{1}\right. & \left.D T_{1}\right\rangle
\end{array} S_{1}\right\rangle \\
& x_{8}=c_{4}\left\langle D \phi_{1}\left\langle c_{1} T_{1} M_{6} w_{1}\right\rangle D S_{1}\right\rangle \\
& c_{1}=D^{2}-a^{2}, c_{2}=M_{1}\left(1+M_{5}\right), c_{3}=1+M_{4}+\left(M_{4} / M_{5}\right) \text {, } \\
& c_{4}=\left(M_{5} / M_{6}\right) \text { and } c_{5}=D^{2}-a^{2} M_{3} \text {. }
\end{aligned}
$$

where $\langle u, v\rangle=\int_{-1 / 2}^{1 / 2} u v d z$ and $w_{1}, T_{1}, \phi_{1}$ and $S_{1}$ are trial functions that satisfy the boundary conditions. The above choice of trigonometry function tacitly implies the use of a higher order Galerkin method. For very large $M_{1}$, one gets the results for the magnetic mechanism, and the critical thermomagnetic Rayleigh number for stationary mode is calculated using

$$
N_{s c}=M_{1} R_{s c}=\frac{x_{1}-a^{2} R_{S}\left(x_{2} S_{T}+x_{3}\right)}{\left(1+M_{5}\right)\left[\left(1-S_{T}\right) \tau\left(x_{5}+x_{7}\right)+x_{6} S_{T}+x_{8}\right]}
$$

\section{DISCUSSION OF RESULTS}

The effect of temperature dependent viscosity on Soret driven thermohaline convection in a ferromagnetic fluid layer heated from below and salted from above saturating an anisotropic porous medium subjected to a transverse uniform magnetic field has been considered by using the Brinkman model and the linear stability analysis. The small thermal perturbation technique is used and normal mode technique is applied for the perturbation quantities. Here, the free-free boundary conditions are used. The present investigation is carried out through stationary instability.

Before we discuss the important results of the system, we turn our attention to the possible range of values of different parameters arising in the study. The range of values of the temperature dependent viscosity parameter $V$ is assumed from 0.1 to 0.5 [12]. The value of anisotropic effect is considered from 0.03 to 3.1 [22]. The buoyancy magnetization parameter $M_{1}$, is assumed to be 1000 [6]. For these type of fluids $M_{2}$ will have a negligible value and hence taken to be zero. The range of Salinity Rayleigh number $R_{\mathrm{S}}$ is between -500 and 500 and Soret parameter $S_{\mathrm{T}}$ ranges from -0.002 to 0.002 [22]. The Brinkman model has been used for permeability $k$ ranges from 0.1 to 0.9 [20] and the non-buoyancy magnetization parameter $M_{3}$ is taken to have from 5 to 25 [22]. The Prandtl number $P_{\mathrm{r}}$ is taken to be 0.01 [20] and the magnetic number $M_{4}, M_{5}$ and $M_{6}$ are taken to be 0.1 [18]. The ratio of mass transport to heat transport $\tau$ is assumed from 0.03 to 0.011 [20].

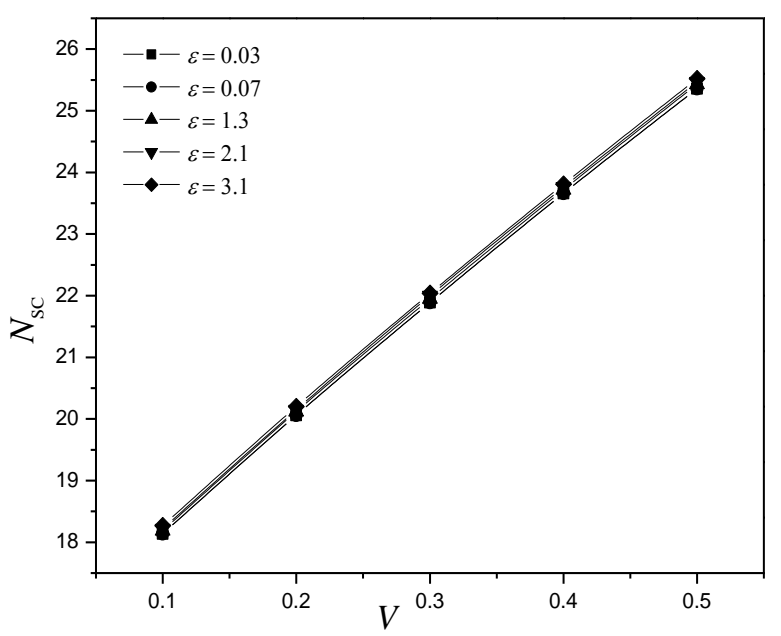

Figure 1. Marginal instability curve for variation of $N_{\mathrm{sc}}$ versus $V$ for various values of anisotropy effect $\varepsilon, R_{\mathrm{S}}=-500$, $S_{\mathrm{T}}=-0.002, k=0.1, M_{3}=5$ and $\tau=0.03$ 
Figure 1 gives the critical magnetic thermal Rayleigh number $N_{\mathrm{sc}}$ versus the temperature dependent viscosity $V$ for different values of an anisotropy parameter $\varepsilon$. It is observed from Figure 1 that the temperature dependent viscosity $V$ has a stabilizing on the system when $V$ increases, $N_{\text {sc }}$ increases and this stabilizing effect of $V$ is much pronounced. Figure 2 represents the plots of critical magnetic thermal Rayleigh number $N_{\text {sc }}$ with respect to non-buoyancy magnetization effect $M_{3}$ for various values of temperature dependent viscosity $V, S_{\mathrm{T}}$ $=-0.002, R_{\mathrm{S}}=-500, \tau=0.05, \varepsilon=0.03$ and $k=0.1$. When $M_{3}$ and $V$ increases, $N_{\text {sc }}$ gets decreasing values. Therefore, the convective system has a destabilizing effect, which is not much pronounced. This is because variation in magnetization releases extra energy which adds up to the thermal energy to destabilize the system.

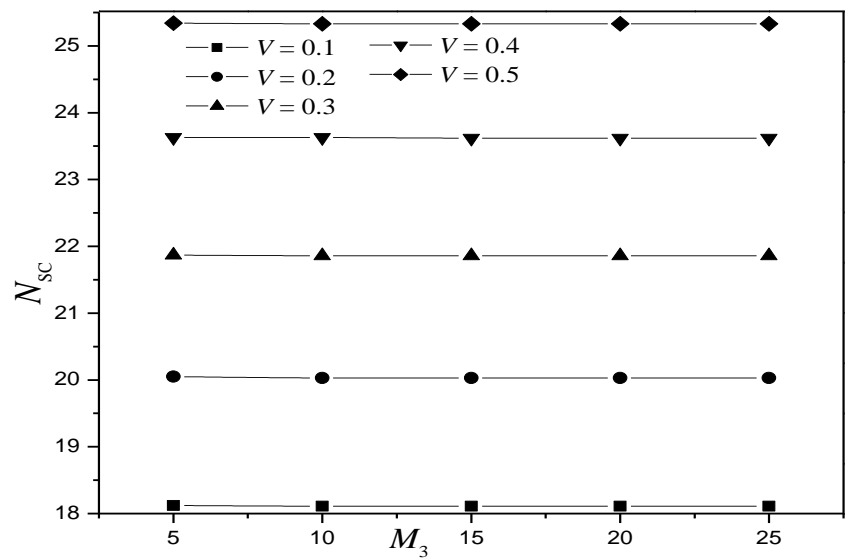

Figure 2. Marginal instability curve for variation of $N_{\mathrm{sc}}$ versus $M_{3}$ for various values of temperature dependent viscosity effect $V, \varepsilon=0.03, R_{\mathrm{S}}=-500, S_{\mathrm{T}}=-0.002, k=0.1$ and $\tau=0.03$

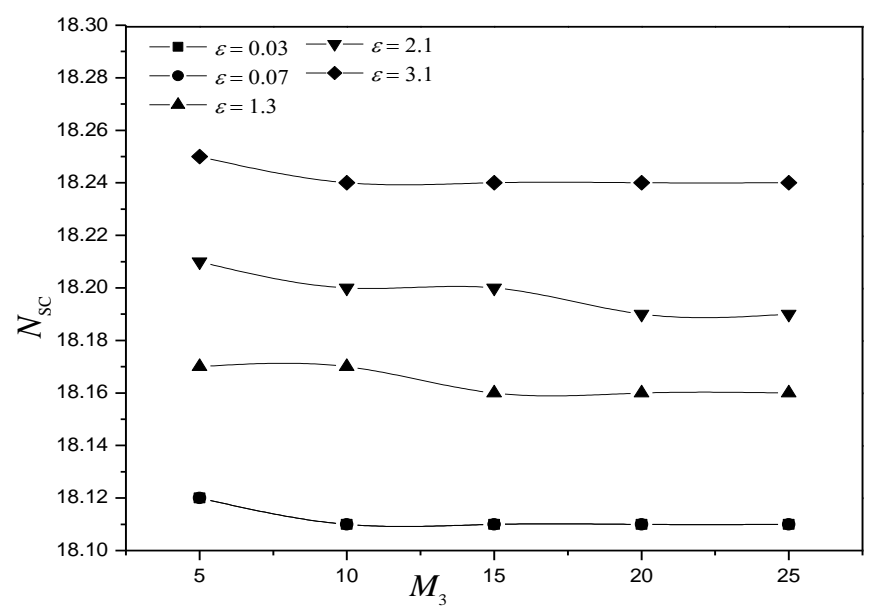

Figure 3. Marginal instability curve for variation of $N_{\mathrm{sc}}$ versus $M_{3}$ for various values of anisotropy effect $\varepsilon, R_{\mathrm{S}}=$ $500, S_{\mathrm{T}}=-0.002, k=0.1, V=0.1$ and $\tau=0.03$

In figure 3, the varation of $N_{\mathrm{sc}}$ versus $M_{3}$ for different values of anisotropy effect of $\mathcal{E}, S_{\mathrm{T}}=-0.002, R_{\mathrm{S}}=-500, \tau=0.05$ and $k=0.1$. It gives that the non-buoyancy magnetization parameter $M_{3}$ has a destabilizing behavior. Also, in the value $\varepsilon=0.03$ and 0.07 , the $N_{\text {sc }}$ gets an exact same effect on the convective system. There is no much variations in $N_{\mathrm{sc}}$ due to the increasing values of $M_{3}$ and ${ }^{\varepsilon}$, which is depicted in figure 3 . But, there are variations in $N_{\mathrm{sc}}$ due to the increasing values of $M_{3}$ and $V$, which is depicted in figure 2. It seems that $M_{3}$ has little effect on the stability. Figure 4 represents the plots of $N_{\mathrm{sc}}$ versus the salinity Rayleigh number $R_{\mathrm{S}}$ for different values of temperature dependent viscosity $V, \quad S_{\mathrm{T}}=-0.002$, $\tau=0.05, \varepsilon=0.03$ and $k=0.1$. It shows that the salinity Rayleigh number $R_{\mathrm{S}}$ has a stabilizing behavior on the convective system. The increasing effect of salt on the system, the system gets more thermal energy and it has stabilizing effect.

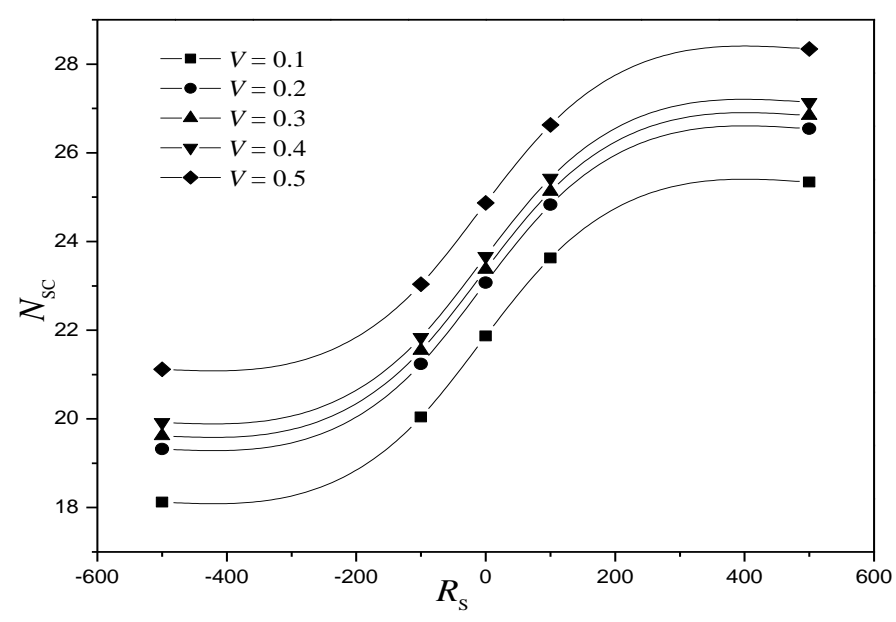

Figure 4. Marginal instability curve for variation of $N_{\mathrm{sc}}$ versus $R_{\mathrm{S}}$ for various values of temperature dependent viscosity effect $V, \varepsilon=0.03, M_{3}=5, S_{\mathrm{T}}=-0.002, k=0.1$ and $\tau=0.03$

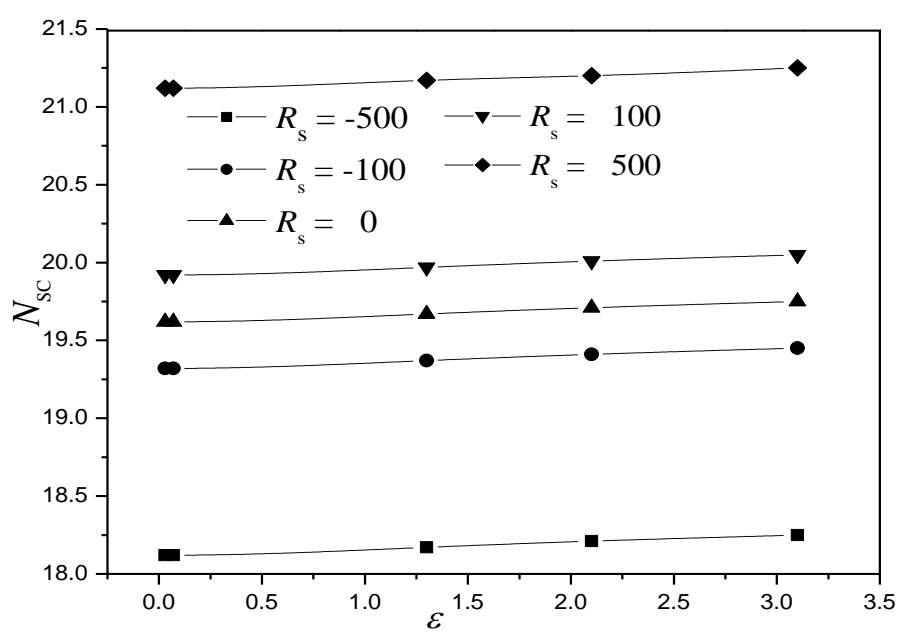

Figure 5. Marginal instability curve for variation of $N_{\mathrm{sc}}$

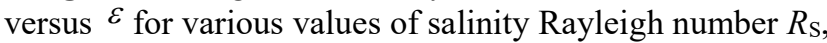
$S_{\mathrm{T}}=-0.002, k=0.1, M_{3}=5, V=0.1$, and $\tau=0.03$

In figure 5 , the variation of $N_{\mathrm{sc}}$ versus $\varepsilon$ for different salinity Rayleigh number $R_{\mathrm{S}}$ is investigated. This figure exhibits a stabilizing effect is not much pronounced because the presence of salinity Rayleigh number $R_{\mathrm{S}}$ increase from 500 to $500, N_{\mathrm{sc}}$ increases. Figure 6 indicates the variation of $N_{\text {sc }}$ versus the interdiffusion of heat and mass, namely Soret effect $S_{\mathrm{T}}$ for different $V$. This figure gives as increase of $S_{\mathrm{T}}$, increase of $N_{\mathrm{sc}}$. This leads to stabilizing effect is much pronounced. But, introducing and increasing of $\varepsilon$ on the 
convective system has a stabilizing behavior is not pronounced much which is depicted in figure 7.

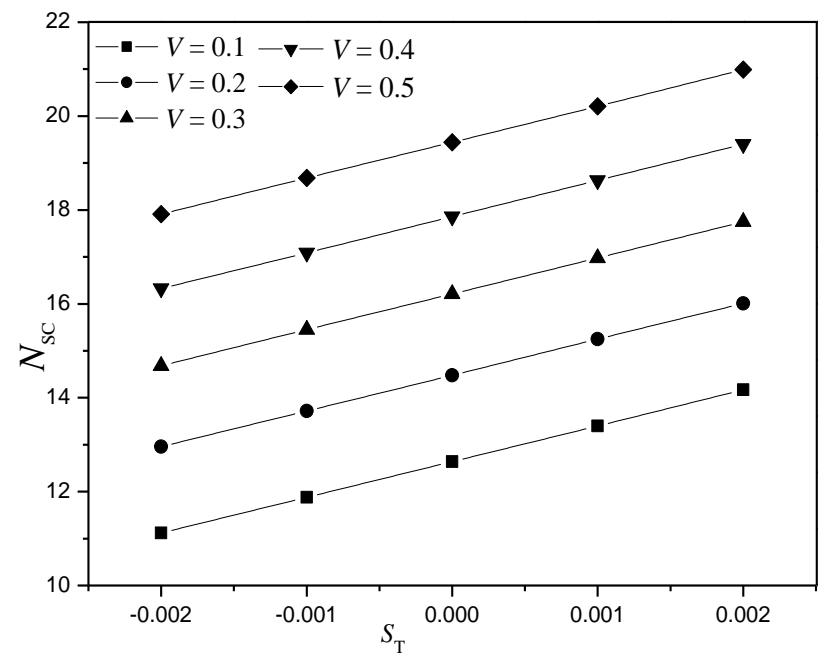

Figure 6. Marginal instability curve for variation of $N_{\mathrm{sc}}$ versus $S_{\mathrm{T}}$ for various values of temperature dependent viscosity effect $V, \varepsilon=0.03, M_{3}=5, S_{\mathrm{T}}=-0.002, k=0.1$ and $\tau=0.03$

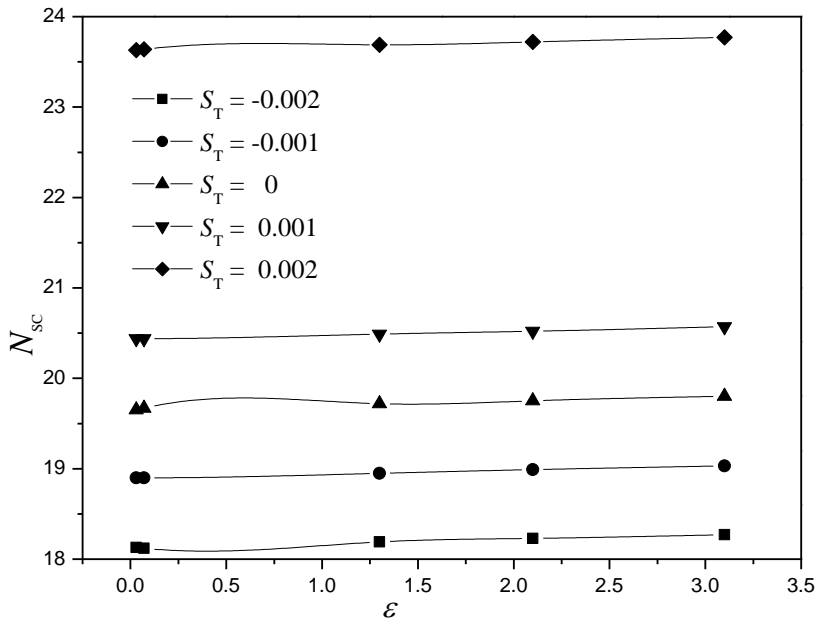

Figure 7. Marginal instability curve for variation of $N_{\mathrm{sc}}$ versus $\varepsilon$ for various values of Soret coefficient $S_{\mathrm{T}}, R_{\mathrm{S}}=$. $500, k=0.1, M_{3}=5, V=0.1$ and $\tau=0.03$

It is observed from figure 8 that the increase in the ratio of mass transport to the heat transport $\tau$ shows a uniform stabilizing behavior, for increasing value of $V$ and $\varepsilon, S_{\mathrm{T}}=$ $0.002, R_{\mathrm{S}}=-500, \tau=0.05$ and $k=0.1$. In this figure, the system is analyzed for stabilizing effect and when increasing of $\mathcal{E}$ from 0.03 to 3.1 , the $N_{\mathrm{sc}}$ has same effect. This is because the increase in mass transport adds up to the system to be top heavy. Figure 9 illustrates that $N_{\mathrm{sc}}$ versus $V$ for different values of permeability of the porous medium $k$. When increasing of $V$ from 0.1 to 0.5 and k from 0.1 to $0.9, N_{\text {sc }}$ is increased. It seems that the system stabilization and which is plotted form positive value of $R_{\mathrm{S}}(=500)$ and $S_{\mathrm{T}}(=0.002)$ and negative range of $R_{\mathrm{S}}(=-500)$ and $S_{\mathrm{T}}(=-0.002)$, there is no change on the situation of the system. It is also observed from the figures that the increase in pore size make the fluid flow easy to cause convection early.

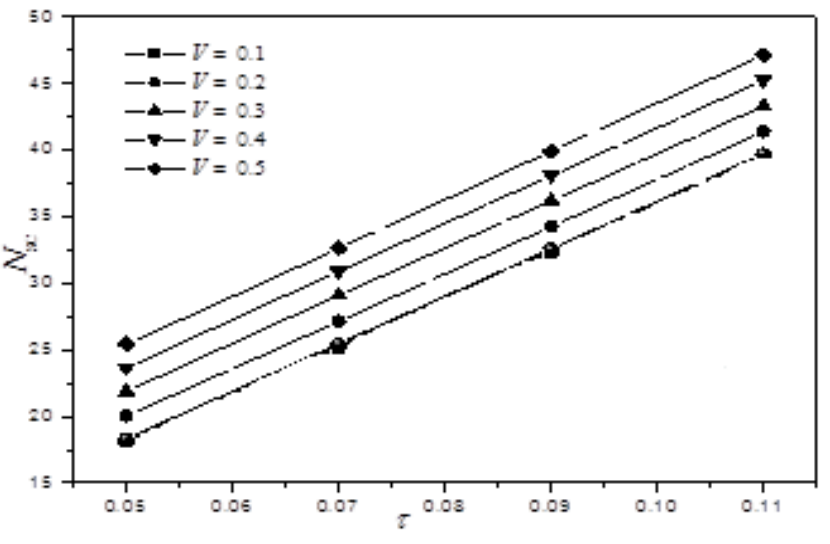

Figure 8. Marginal instability curve for variation of $N_{\mathrm{sc}}$ versus ${ }^{\tau}$ for various values of $V, S_{\mathrm{T}}=-0.002, R_{\mathrm{S}}=-500, k=$ $0.1, \varepsilon=0.03$ and $M_{3}=5$

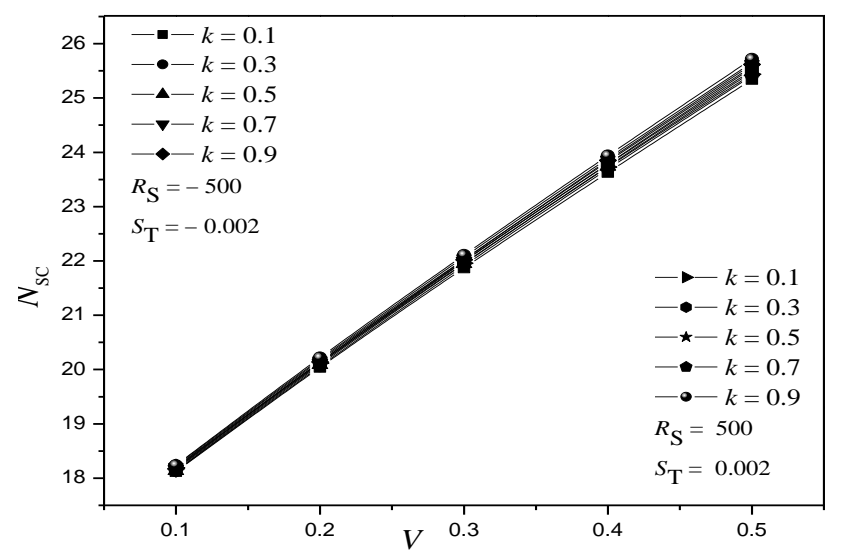

Figure 9. Marginal instability curve for variation of $N_{\mathrm{sc}}$ versus $V$ for various values of $k\left(R_{\mathrm{S}}=-500, S_{\mathrm{T}}=-0.002\right.$ and $\left.R_{\mathrm{S}}=500, S_{\mathrm{T}}=0.002\right),{ }^{\varepsilon}=0.03, M_{3}=5$ and $\tau=0.03$

\section{CONCLUSION}

Ferro thermoconvective instability of magnetic fluid layer heated from below and salted from above in the presence of an anisotropic porous medium and temperature field dependent viscosity suspended to a transverse uniform magnetic field has been investigated using Brinkman model with Soret effect. The computational Galerkin method is used. In this analysis, we have analyzed the effect of various parameters like medium permeability $k$, anisotropic parameter $\varepsilon$, ratio of mass transport to heat transport $\tau$, non- buoyancy magnetization parameter $M_{3}$, temperature dependent viscosity parameter $V$, Soret coefficient $S_{\mathrm{T}}$ and Salinity Rayleigh number $R_{\mathrm{S}}$.

The destabilizing behavior is analyzed for non-buoyancy magnetization parameter $M_{3}$ on the convective system. The stabilizing effect is investigated for the temperature dependent viscosity parameter $V$ in a very small and large value of salinity concentration, which is depicted in figure 9. In this moment, the system gets slight variation in convection process. Moreover, in some investigations, the porous medium $k$ and anisotropy effect $\varepsilon$ are analyzed for destabilizing effect [22]. But the introduction of temperature dependent viscosity $V$, the permeability of the porous medium $k$ and anisotropy effect $\varepsilon$ have a stabilizing effect. 
Thus, from above analysis, one can conclude that the nonbuoyancy magnetization parameter, temperature gradient, porous medium, anisotropic porous medium and salinity gradient have a profound influence on the onset of convection.

\section{ACKNOWLEDGEMENT}

The author K. Raju is grateful to Dr. J. Arawindhan (Director) and Dr. Nirmalkumar (Principal), Achariya Arts and Science College, Villianur, Puducherry for their constant encouragement.

\section{REFERENCES}

[1] Berkovsky B, Bastovoy V. (1996). Magnetic Fluids and Application Handbook. Begell House Publishers, New York.

[2] Gazeau F, Baravian C, Bacri JC, Perzynski P, Shiomis M.I. (1997). Energy conversion in ferrofluids: Magnetic nanoparticles as motors or generators. Physics Reviews - E 56: 614. https://doi.org/10.1103/PhysRevE.56.614

[3] Odenbach S, Thurm S. (2012). Magnetoviscous effects in ferrofluids. eStefan Odenbach, Springer-Verlag, Berlin, Heidelberg.

[4] Rosensweig RE. (1985). Ferro hydrodynamics, Cambridge University Press, Cambridge.

[5] Chandrasekhar S. (1981). Hydrodynamics and Hydromagnetic stability. Dover Publication, New York.

[6] Finlayson BA. (1970). Convective instability of ferromagnetic fluids. International Journal of Fluid Mechanics 40: $753-767$ https://doi.org/10.1017/S0022112070000423

[7] Schwab L, Hildebrandt U, Stierstadt K. (1983). Magnetic Bénard convection. Journal of Magnetism and Magnetic Materials 39: 113-114. https://doi.org/10.1016/03048853(83)90412-2

[8] Stiles PJ, Kagan M. (1990). Thermoconvective instability of a horizontal layer of ferrofluid in a strong vertical magnetic field. Journal of Magnetism and Magnetic Materials 85: 196-198. https://doi.org/10.1016/0304-8853(90)90050-Z

[9] Ursino N, Roth K, Gimmi T, Flühler H. (2000). Upscaling of anisotropic in unsaturated Miller-similar porous media. Water Resources Research 36: 421-430. https://doi.org/10.1029/1999WR900320

[10] Epherre JF. (1975). Critere d' apparition de la convection naturelle dans une couche poreuse anisotrope. Revue Générale de Thermique 168: 949 - 950.

[11] Vaidyanathan G, Sekar R, Balasubramanian R. (1991). Ferroconvective instability of fluids saturating a porous medium. International Journal of Engineering Sciences 29: $\quad 1259-1267 . \quad$ https://doi.org/10.1016/00207225(91)90029-3

[12] Ramanathan A, Muchikel N. (2006). Effect of temperature dependent viscosity on ferroconvection in a porous medium. International Journal of Applied Mechanics and Engineering 11: 93-104.

[13] Suresh G, Vasanthakumari R, Radja P. (2012). Numerical study on the effect of temperature dependent viscosity on ferroconvection in an anisotropic porous medium. International Journal of Engineering Technology and Advanced Engineering 2: 51-55.
[14] Nanjundappa CE, Shivakumara IS, Arunkumar R. (2010). Bénard-Marangoni ferroconvection magnetic field dependent viscosity. Journal of Magnetism and Magnetic Materials. 322: 2256-2263. https://doi.org/10.1016/j.jmmm.2010.02.021

[15] Nanjundappa CE, Shivakumara I., Arunkumar R. (2013). Onset of Marangoni-Bénard ferroconvection with temperature dependent viscosity. Microgravity Sci. Technol 25: 103-112. https://doi.org/10.1007/s12217012-9330-9.

[16] Siddheshwar PG. (2004). Thermorheological effect on magneto convection in weak electrically conducting fluids and $1 \mathrm{~g}$ or $\mu g$, Pramana J. Phy 62: 61-68. https://doi.org/10.1007/BF02704425

[17] Baines PG, Gill SE. (1969). On thermohaline convection with linear gradients. Journal of Fluid Mechanics 37: 289-306. https://doi.org/10.1017/S0022112069000553

[18] Vaidyanathan G, Sekar R, Ramanathan A. (1995). Ferrothermohaline convection in a porous medium. Journal of Magnetism and Magnetic Materials 149: 137142. https://doi.org/10.1016/0304-8853(95)00356-8

[19] Vaidyanathan G, Sekar R, Ramanathan A. (1997). Ferrothermohaline convection. Journal of Magnetism and Magnetic Materials 176: 321-330. https://doi.org/10.1016/S0304-8853(97)00468-X

[20] Vaidyanathan G, Sekar R, Hemalatha R, Vasanthakumari R, Senthilnathan S. (2005). Soretdriven ferro thermohaline convection. Journal of Magnetism and Magnetic Materials 288: 460-469. https://doi.org/10.1016/j.jmmm.2004.09.137

[21] Sekar R, Vaidyanathan G, Hemalatha R, Senthilnathan S. (2006). Effect of sparse distribution pores in a Soretdriven ferro thermohaline convection. Journal of Magnetism and Magnetic Materials 302: 20-28. https://doi.org/10.1016/j.jmmm.2005.08.008

[22] Sekar R, Raju K, Vasanthakumari R. (2013). A linear analytical study on Soret-driven ferrothermohaline convection in an anisotropic porous medium. Journal of Magnetism and Magnetic Materials 331: 122-128. https://doi.org/10.1016/j.jmmm.2012.10.028

[23] Sekar R, Raju K, Vasanthakumari R. (2013). Linear stability analysis of coriolis force on ferrothermohalineconvection saturating an anisotropic porous medium with Soret effect. Global Journal of $\begin{array}{lll}\text { Mathematical } \quad \text { Analysis } & \text { 1(2): }\end{array}$ https://doi.org/10.14419/gjma. v1i2.858

[24] Sekar R, Raju K. (2013). Effect of magnetic field dependent viscosity on Soret-driven thermoconvective instability of ferromagnetic fluid in the presence of rotating anisotropic porous medium of sparse particle suspension. International Journal of Mathematical Sciences 12: 13-31.

\section{NOMENCLATURE}
B Magnetic induction (T)
$\mathrm{C}_{\mathrm{vH}} \quad$ Effective heat capacity at constant volume and magnetic field $\left(\mathrm{kJm}^{-3} \mathrm{~K}^{-1}\right)$
d Thickness of the fluid layer (m)
D/Dt Convective derivative [ $D / D t=\partial / \partial t+\vec{q} \cdot \nabla](\mathrm{s}$ 1)
g Gravitational acceleration $(0,0-\mathrm{g})\left(\mathrm{ms}^{-2}\right)$ 
$\mathrm{H} \quad$ Magnetic field $\left(\mathrm{Am}^{-1}\right)$

$k_{0} \quad$ Resultant wave number $\left[k_{\mathrm{o}}=\sqrt{k_{x}^{2}+k_{y}^{2}}\right]\left(\mathrm{m}^{-1}\right)$

$\mathrm{k}_{1} \quad$ Permeability of the porous medium

$\mathrm{K}_{1} \quad$ Thermal diffusivity $\left(\mathrm{Wm}^{-1} \mathrm{~K}^{-1}\right)$

$\mathrm{K}_{2} \quad$ Salinity magnetic coefficient [ $\equiv(\partial \mathrm{M} / \partial S)_{H_{0}, T_{0}}$ ]

$\mathrm{kx}, \mathrm{k}_{\mathrm{y}} \quad$ Wave number in the $\mathrm{x}$ and $\mathrm{y}$ direction $\mathrm{m}^{-1}$

$\mathrm{K}_{\mathrm{s}} \quad$ Concentration diffusivity $\left(\mathrm{Wm}^{-1} \mathrm{k}^{-1} \mathrm{~g}^{-1}\right)$

$\mathrm{k} \quad$ Unit vector in vertical direction

$\mathrm{K} \quad$ Pyromagnetic coefficient [ $\left.\equiv-(\partial \mathrm{M} / \partial T)_{H_{0}, T_{0}}\right]$

M Magnetization $\left(\mathrm{Am}^{-1}\right)$

$\mathrm{M}_{0} \quad$ Mean value of the magnetization at $\mathrm{H}=\mathrm{H}_{0}$ and $\mathrm{T}$ $=\mathrm{T}_{0}$.

$\mathrm{P} \quad$ Hydrodynamic pressure $\left(\mathrm{Nm}^{-2}\right)$

q Velocity of the ferrofluid ( $u$

$\mathrm{v} \quad \mathrm{w})\left(\mathrm{ms}^{-1}\right)$

$\mathrm{S} \quad$ Solute concentration $(\mathrm{kg})$

$\mathrm{S}_{0} \quad$ Average salinity

$\mathrm{S}_{\mathrm{T}} \quad$ Soret coefficient

$\mathrm{T} \quad$ Temperature $(\mathrm{K})$

$\mathrm{T} \quad$ Time (s)

$\mathrm{T}_{0} \quad$ Average temperature

$\varepsilon \quad$ Anisotropy parameter $\alpha_{t} \quad$ Coefficient of thermal expansion $\left(\mathrm{K}^{-1}\right)$

$\alpha_{s} \quad$ Solvent coefficient of expansion $\left(\mathrm{K}^{-1}\right)$

$\beta_{t} \quad$ Uniform temperature gradient $\left(\mathrm{Km}^{-1}\right)$

$\beta_{s} \quad$ Uniform concentration gradient $\left(\mathrm{kgm}^{-1}\right)$

$\delta \quad$ Small positive quantity

$\mu_{0} \quad$ Magnetic permeability of vacuum $\left(\mathrm{NA}^{-2}\right)$

$\mu_{1} \quad$ Reference viscosity at $\mathrm{T}=\mathrm{T}_{0}$

$\mu \quad$ Dynamic viscosity $\left(\mathrm{kgm}^{-1} \mathrm{~s}^{-2}\right)$

$\rho_{0} \quad$ Mean density of the clean fluid $\left(\mathrm{kgm}^{-3}\right)$

$\rho \quad$ Density of the fluid $\left(\mathrm{kgm}^{-3}\right)$

$\sigma \quad$ Growth rate $\left(\mathrm{s}^{-1}\right)$

$\varphi \quad$ Viscous dissipation factor containing second order terms in velocity

$\phi \quad$ Magnetic scalar potential (A)

$\theta \quad$ Perturbation in temperature (K)

$\chi \quad$ Magnetic susceptibility [ $\left.\equiv(\partial \mathrm{M} / \partial H)_{H_{0}, T_{0}}\right]$

$\nabla \quad$ Vector different operator $[\equiv \overrightarrow{\mathrm{i}}(\partial / \partial x)+\vec{j}(\partial / \partial y)+\vec{k}(\partial / \partial z)]$ 